\title{
Impact Factors on Posterior Modified Transfacet Debridement for Thoracic Spinal Tuberculosis Basing on Regression and Classification Analysis
}

\author{
Xiaoping Chen $\mathbb{D},{ }^{1}$ Jiamin Lin, ${ }^{1}$ Han Huang, ${ }^{2}$ and Yunpeng Huang $\mathbb{D}^{3}$ \\ ${ }^{1}$ Department of Statistics, College of Mathematics and Informatics \& FJKLMAA, Fujian Normal University, \\ Fuzhou 350117, China \\ ${ }^{2}$ Department of Statistics and Data Science, Southern University of Science and Technology, Shenzhen 518055, China \\ ${ }^{3}$ Department of Spine Surgery, The First Affiliated Hospital of Fujian Medical University, Fuzhou 350005, China
}

Correspondence should be addressed to Yunpeng Huang; yawn7770@126.com

Received 15 April 2020; Revised 17 June 2020; Accepted 7 July 2020; Published 1 August 2020

Academic Editor: Chenxi Huang

Copyright ( $\odot 2020$ Xiaoping Chen et al. This is an open access article distributed under the Creative Commons Attribution License, which permits unrestricted use, distribution, and reproduction in any medium, provided the original work is properly cited.

\begin{abstract}
Posterior transfacet approach has been proved to be a safe and effective access to treat thoracic disc herniation. However, the influencing factors of posterior modified transarticular debridement for thoracic tuberculosis have not been reported in the clinical literature. From 2009 to 2014, 37 patients with TST underwent a posterior modified transfacet debridement, interbody fusion following posterior instrumentation, under the cover of 18 months of antituberculosis chemotherapy. The patients were evaluated preoperatively and postoperatively in terms of Frankel Grade, visual analog scale (VAS) pain score, kyphotic Cobb angle, and bone fusion. Blood loss (positive correlation) and focal debridement (positive correlation) could affect operative time. Operative time (positive correlation) could affect blood loss. While, age (positive correlation), PostE (negative correlation), and T_FocalDebridement (positive correlation) could affect bone fusion. The accuracy of naive bayes classifier model is $86.11 \%$. Our preliminary results show that blood loss and focal debridement could affect operative time; operative time could affect blood loss; age, PostE, and T_FocalDebridement could affect bone fusion; the naive Bayes classifier model can predict the KirkaldyWillis accurately.
\end{abstract}

\section{Introduction}

Tuberculosis (TB), an infectious disease caused by mycobacterium tuberculosis (MTB), is the ninth leading cause of global death, ranking above acquired immune deficiency syndrome (AIDS). The spine is the most frequently involved part of extrapulmonary tuberculosis, which is more common in thoracolumbar vertebrae, $30.3 \%$ to $55.8 \%$ of which are thoracic tuberculosis and about $67 \%$ of thoracic tuberculosis patients are accompanied by tuberculosis $[1,2]$. Vertebral body destruction will lead to spinal instability, spinal cord damage, and deformity. Therefore, in addition to standardized antituberculosis treatment, surgery is also an important treatment method for thoracic tuberculosis treatment [3].
As an important surgical intervention in the treatment of spinal tuberculosis, surgical treatment aims to effectively remove the lesion, completely relieve the neurospinal compression, restore the height of the affected vertebra as far as possible, and rebuild the stability of the spine. After continuous exploration and development, the current surgical treatment methods are classified according to the approach, mainly including anterior approach, posterior approach, and combined approach. (1) Anterior approach: compared with other methods, anterior approach is the most convenient method for removing lesions because it can directly reach the lesion and has a good operating field of vision [4]. (2) Combined approach: both lesion clearance and deformity correction. However, anterior or anterior combined approaches have a higher rate of primary activity 
and mortality [5, 6]. (3) Posterior approach: posterior approaches such as posterolateral, pedicle, or posterior approaches and their variants have been proven to be a safe and effective method for the treatment of thoracic disc herniation (TDH) with a relatively low incidence. In addition, posterior approach can better correct kyphosis, which is the most common complication of TST [7]. Some studies believed that, in terms of operation time, the combined approach was longer than the anterior approach, but there was no statistical difference with the posterior approach. In terms of blood loss, anterior and posterior combined approaches were more common than anterior or posterior approaches. There was no clinical literature to report the influencing factors of posterior modified transarticular debridement for thoracic tuberculosis. This paper considers the influencing factors of operative time, blood loss, bone fusion, and the classification of the KirkaldyWillis.

\section{Methods}

Between 2009 and 2014, the authors treated 37 consecutive patients with TST via a modified transfacet approach [7]. This study was approved by the Ethics Committee of Affiliated Hospital of Fujian Medical University.

2.1. Preoperative Surgery. Patients were treated with standard chemotherapy regimen of isoniazid $(\mathrm{H})$, rifampicin (R), ethambutol (E), and pyrazinamide (Z) (HREZ) at least 4 weeks before surgery, including isoniazid $(300 \mathrm{mg} / \mathrm{d})$, rifampicin $(450 \mathrm{mg} / \mathrm{d})$, ethambutol $(750 \mathrm{mg} / \mathrm{d})$, and pyrazinamide $(750 \mathrm{mg} / \mathrm{d})$. ESR was $44.7 \pm 23.3 \mathrm{~mm} / \mathrm{h}$. And the mean kyphosis angle was $29.4 \pm 10.9^{\circ}$. Surgery is performed when ESR is significantly reduced $(<40 \mathrm{~mm} / \mathrm{h})$. Preoperative antituberculosis treatment can reduce mycobacterium tuberculosis in focus and improve surgical safety. Bony spinal fusion was assessed according to the criteria defined by Lee et al. [8], which was graded as shown in Table 1. And in our study, all patients obtained definitive bone fusion.

2.2. Statistical Principle. In order to examine the factors that might affect operative time, the multivariable linear regression model is adopted. Operative time is considered as a continuous and dependent variable, whereas blood loss, PreK, FFUK, and focal debridement are constituted the independent variables. The multivariable linear regression model is also utilized for investigating whether operative time could affect blood loss. Blood loss is considered as a constant and dependent variable, whereas operative time, bone fusion, and PreK constituted the independent variables. The naive Bayes classifier model is also utilized for investigating what affects the KirkaldyWillis grade. All the statistical analyses were performed using the $\mathrm{R}$ Project (version 3.5.1, 2018, The $\mathrm{R}$ Foundation for Statistical Computing), the $\mathrm{R}$ package e1071, corrplot needs to be loaded. The results were considered statistically significant at $p<0.05$.

Multiple Linear Regression [9] attempts to find a linear equation with known data to describe the relationship between two or more independent variables and dependent variable and use this linear equation to predict the results.

Let the dependent variable be $Y$, and the $k$ independent variables affecting the dependent variable be $X_{1}, X_{2}, \ldots, X_{K}$, respectively. Let us assume that the influence of each independent variable on the dependent variable $Y$ is linear, that is, under the condition that other independent variables remain unchanged, the mean value of $Y$ changes uniformly with the change of independent variable $X_{i}$ :

$$
Y_{i}=\beta_{i}^{(0)}+\beta_{i}^{(1)} X_{i}^{(1)}+\beta_{i}^{(2)} X_{i}^{(2)}+\cdots+\beta_{i}^{(K)} X_{i}^{(K)}+\varepsilon .
$$

This is called the population regression model, and $\beta_{i}^{(0)}, \beta_{i}^{(1)}, \ldots, \beta_{i}^{(K)}$ are the regression parameters.

The principle of naive Bayes classifier [10] is a statistical method. Bayes theorem is the theoretical basis of classifier modeling. It uses conditional probability principle, prior information, and sample data information to determine the probability of event occurrence. In addition, naive Bayes model is a kind of generating model, which directly models the joint probability to obtain the target probability. The algorithm is as follows.

$T=\left\{\left(x_{1}, y_{1}\right),\left(x_{2}, y_{2}\right), \ldots,\left(x_{n}, y_{n}\right)\right\}$, among them $x_{i}=$ $\left(x_{i}^{(1)}, x_{i}^{(2)}, \ldots, x_{i}^{(K)}\right)^{T}, x_{i}^{(j)}$ is the $j$ th independent variable of the $i$ th sample, $a_{j l}$ is the $j$ th independent variable of the $l$ th value that it is possible to take $j=1,2, \ldots, K$ and $l=1,2, \ldots, S_{j} ; y_{i} \in\left\{c_{1}, c_{2}, \ldots, c_{P}\right\}$.

(1) Calculate the prior probability and conditional probability:

$$
\begin{gathered}
P\left(Y=c_{p}\right)=\frac{\sum_{i=1}^{n} I\left(y_{i}=c_{p}\right)}{n}, \quad p=1,2, \ldots, P, \\
P\left(X^{(j)}=a_{j l} \mid Y=c_{p}\right)=\frac{\sum_{i=1}^{n} I\left(x_{i}^{(j)}=a_{j l}, y_{i}=c_{p}\right)}{\sum_{i=1}^{n} I\left(y_{i}=c_{p}\right)}, \quad j=1,2, \ldots, K ; l=1,2, \ldots, S_{j} ; p=1,2, \ldots, P .
\end{gathered}
$$

(2) For a given sample of $x=\left(x^{(1)}, x^{(2)}, \ldots, x^{(n)}\right)^{T}$, compute

$$
P\left(Y=c_{p}\right) \prod_{j=1}^{K} P\left(X^{(j)}=x^{(j)} \mid Y=c_{p}\right), \quad p=1,2, \ldots, P .
$$


TABLE 1: The modified criteria of Lee et al for radiological fusion.

\begin{tabular}{|c|c|}
\hline Grade & Description \\
\hline Definitive fusion & $\begin{array}{c}\text { Definitive bony trabecular bridging across the graft-host interface, no movement }\left(<3^{\circ}\right) \text { on a flexion-extension } \\
\text { radiograph and no gap at the interface }\end{array}$ \\
\hline Probable fusion & No definitive bony trabecular crossing, but no detectable movement and no identifiable gap at the interface \\
\hline $\begin{array}{l}\text { Possible } \\
\text { pseudarthrosis }\end{array}$ & No bony trabecular crossing, no movement, but identifiable gap at the interface \\
\hline $\begin{array}{l}\text { Definite } \\
\text { pseudarthrosis }\end{array}$ & No traversing trabecular bone, definitive gap, and movement $>3^{\circ}$ \\
\hline
\end{tabular}

(3) Determine the classification of sample $x$ :

$$
y=\arg \max _{c_{p}} P\left(Y=c_{p}\right) \prod_{j=1}^{K} P\left(X^{(j)}=x^{(j)} \mid Y=c_{p}\right) .
$$

\section{Result}

We find that blood loss (positive correlation) and focal debridement (positive correlation) could affect operative time, but not bone fusion, gender, PreA, PostA, FFUA, age, hospital stay, segments, follow-up, PreK, PostK, PreE, PostE, FUUK, FFUE, and T_Level. We also find that operative time (positive correlation) could affect blood loss, but not gender, PreA, PostA, FFUA, age, hospital stay, segments, follow-up, PostK, FUUK, PreK, PreE, PostE, FFUE, T_Level, and T_FocalDebridement. We also find age (positive correlation), PostE (negative correlation), and T_FocalDebridement (positive correlation) could affect bone fusion, but not gender, PreA, PostA, FFUA, hospital stay, segments, followup, PostK, PreE, FUUK, FFUE, and T_Level.

Final equation 1:

Operativetime $=108.27007+0.11575 *$ bloodloss

$$
+23.61903 * \text { T_FocalDebridement. }
$$

As for the influencing factor model of operative time, $F$ test is performed on the whole model (Table 2), its $F$-statistic is 57.76 on 2 and $34 \mathrm{DF}$, and $p$ value is $1.161 e-11$, indicating that the model is significant on the whole, that is, the model is significant highly. The goodness of fit $R^{2}$ is 0.7726 , indicates that the model has a good fitting effect and can better explain the influencing factors of operative time. In patients with the same T_FocalDebridement, operative time increased by an average of 0.11575 if blood loss increases $1 \mathrm{ml}$, its $p$ value is 0.000168 , which indicates that at the significance level of 0.001 , and blood loss has a significant effect on the operative time. Also, operative time increased by an average of 23.61903 as T_FocalDebridement increased by 1 segment when the blood loss is the same, its $p$ value is 0.035237 , which indicates that at the significance level of 0.05, and T_FocalDebridement factor has a significant effect on the operative time.

Final equation 2:

bloodloss $=-506.0542+4.4878 *$ operativetime.

As for the influencing factor model of blood loss, F test is performed on the whole model (Table 3), and its F-statistic is
99.85 on 1 and $35 \mathrm{DF}$, and $p$ value is $8.648 e-12$, indicating that the model is significant highly on the whole, that is, the model is significant. The goodness of fit $R^{2}$ is 0.7404 , indicates that the model has a good fitting effect and can better explain the influencing factors of blood loss. Blood loss increased by an average of 4.4878 if operative time increased $1 \mathrm{~min}$, its $p$ value is $8.65 e-12$, which indicates that at the significance level of 0.001 , and the operative time factor has a significant effect on the blood loss.

Final equation 3:

$$
\begin{aligned}
\text { Bonefusion }= & 2.75352+0.04326 * \text { Age }-0.08810 * \text { PostE } \\
& +1.53847 * \text { T_FocalDebridement. }
\end{aligned}
$$

As for the influencing factor model of bone fusion, $F$ test is performed on the whole model (Table 4), its F-statistic is 13.12 on 3 and $31 \mathrm{DF}$, and $p$ value is $8.342 e-06$, indicating that the model is significant on the whole, that is, the model is significant highly. The goodness of fit $R^{2}$ is 0.5026 , indicates that the model has a good fitting effect and can better explain the influencing factors of bone fusion. In patients with the same PostE and T_FocalDebridement, bone fusion increased by an average of 0.04326 if age increases 1 , its $p$ value is 0.00286 , which indicates that at the significance level of 0.001 , age has a significant effect on the bone fusion. Also, bone fusion decreased by an average of 0.08810 as PostE increased by 1 when the age and T_FocalDebridement are the same, its $p$ value is 0.03415 , which indicates that at the significance level of 0.05 , PostE factor had a significant effect on the bone fusion. Also, bone fusion increased by an average of 1.53847 as T_FocalDebridement increased by 1 when the age and PostE are the same, its $p$ value is $3.99 e-06$ indicates that at the significance level of 0.001 , and T_FocalDebridement factor had a significant effect on the bone fusion.

After deleting one sample in which KirkaldyWillis is two, only the samples of KirkaldyWillis $=3$ and KirkaldyWillis $=4$ are trained. The correlation coefficient matrices of gender, bone fusion, follow-up, FFUK, FFUA, and FFUE are calculated, as shown in Table 5.

As shown in Figure 1, we can find that the interior color of each circle is lighter except the diagonal line, indicating that the correlation coefficient between variables is low and the degree of correlation is not high. The above variables can be used for naive Bayes classification modeling.

The confusion matrix is obtained in Table 6. 
TABle 2: Model summary for operative time.

\begin{tabular}{lccc}
\hline & & Primary independent variables & \\
& Coefficient estimate & Standard error & $p$ value \\
\hline (Intercept) & 108.27007 & 17.71126 & $<0.001^{* * *}$ \\
Blood loss & 0.11575 & 0.02739 & $<0.001^{* * *}$ \\
T_FocalDebridement & 23.61903 & 10.76961 & $0.035^{*}$ \\
\hline Statistic & Value & DF & $p$ value \\
\hline$F$ & 57.76 & $(2,34)$ & $<0.001^{* * *}$ \\
$R^{2}$ & 0.7726 & & \\
\hline
\end{tabular}

TABle 3: Model summary for blood loss.

\begin{tabular}{lccc}
\hline & & Primary independent variables & \\
& Coefficient estimate & Standard error & $p$ value \\
\hline (Intercept) & -506.0542 & 103.9502 & $<0.001$ \\
Operative time & 4.4878 & 0.4491 & $<0.001^{* * *}$ \\
\hline Statistic & Value & DF*** $^{* * *}$ & $p$ value \\
\hline$F$ & 99.85 & $(1,35)$ & $<0.001^{* * *}$ \\
$R^{2}$ & 0.7404 & & \\
\hline
\end{tabular}

TABle 4: Model summary for bone fusion.

\begin{tabular}{lccc}
\hline & Coefficient estimate & Primary independent variables & \\
& Standard error & $p$ value \\
\hline (Intercept) & 2.75352 & 0.97119 & $<0.01^{* *}$ \\
Age & 0.04326 & 0.01342 & $<0.01^{* *}$ \\
PostE & -0.08810 & 0.03986 & $<0.05^{*}$ \\
T_FocalDebridement & 1.53847 & 0.27879 & $<0.001^{* * *}$ \\
\hline Statistic & Value & DF & $p$ value \\
\hline$F$ & 13.12 & $(3,33)$ & $<0.001^{* * *}$ \\
$R^{2}$ & 0.5026 & & \\
\hline
\end{tabular}

TABle 5: Sample correlation matrix.

\begin{tabular}{|c|c|c|c|c|c|c|}
\hline & Gender & Bone fusion & Follow-up & FFUK & FFUA & FFUE \\
\hline Gender & 1.0000000 & -0.2805976 & -0.1155847 & 0.0715071 & -0.1428571 & 0.3157139 \\
\hline Bone fusion & -0.2805976 & 1.0000000 & 0.2229494 & 0.2665206 & -0.1624512 & -0.2556321 \\
\hline Follow-up & -0.1155847 & 0.2229494 & 1.0000000 & 0.3185323 & 0.0761168 & -0.0372737 \\
\hline FFUK & 0.0715071 & 0.2665206 & 0.3185323 & 1.0000000 & -0.0449019 & -0.0057099 \\
\hline FFUA & -0.1428571 & -0.1624512 & 0.0761168 & -0.0449019 & 1.0000000 & -0.1733689 \\
\hline FFUE & 0.3157139 & -0.2556321 & -0.0372737 & -0.0057099 & -0.1733689 & 1.0000000 \\
\hline
\end{tabular}

Because naive Bayes model is a kind of generating model, it directly models the joint probability to obtain the target probability. Through modeling, we can only get the classification probability of each sample, so as to determine the category, that is, no explicit model can be obtained. The classification accuracy is $86.11 \%$, which indicates the percentage of all correctly predicted samples to all samples; recall is $96.6 \%$, which indicates the correct percentage of all samples predicted with KirkaldyWillis $=4$; precision is $87.5 \%$, which indicates the proportion of all KirkaldyWillis samples predicted to be KirkaldyWillis $=4$.

\section{Discussion}

Operative data of 37 patients with TST were recorded with multiple indicators of the operative level. Multiple linear regression is also suitable for analyzing the influence of multiple surgical factors on one operative variable. The number of vertebral segments debridement is the most important factor affecting the operation time. When other surgical factors remain unchanged, the greater the amount of blood loss, the longer the operation time. Bone fusion time is the most important factor affecting blood loss. When other surgical factors remain unchanged, the longer the operation time, the longer the bone fusion time or the greater the preoperative the KirkaldyWillis score, the greater the blood loss. And both models have high $R^{2}$ values. The posterior transfacet-modified approach has been reported to be a safe and effective method for the treatment of thoracic spinal tuberculosis (TST) with low morbidity in the precious report [7]. However, the impact factors of the surgical approach for TST have not been intensively analyzed. Multiple linear regressions are indeed applicable for analyzing the impact of multiple factors on one operative variable. The aim 


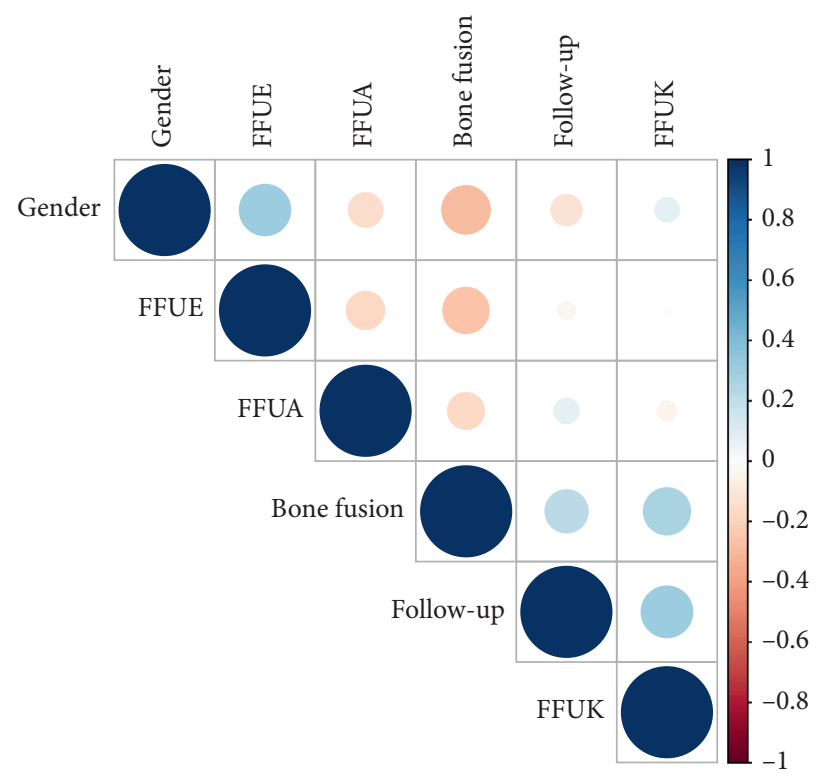

FIgURE 1: Sample correlation diagram.

TABLE 6: Confusion matrix.

\begin{tabular}{lccc}
\hline & & \multicolumn{2}{c}{ Predicted } \\
& & KirkaldyWillis $=4$ & KirkaldyWillis $=3$ \\
\hline \multirow{2}{*}{ Actual } & KirkaldyWillis $=4$ & 3 & 4 \\
& KirkaldyWillis $=3$ & 1 & 28 \\
\hline
\end{tabular}

of this study was to validate the efficacy and safety of posterior modified transfacet surgical approach for the treatment of TST by using multiple linear regressions. Naive Bayes classifier can classify category data well, with high recall and accuracy. On the whole, the analysis results are consistent with clinical results. Because naive Bayes is sensitive to sample equilibrium, samples with KirkaldyWillis $=2$ is deleted before the classification of KirkaldyWillis, so we cannot judge the samples with KirkaldyWillis $=2$, which needs to be improved. Machine learning classification methods, such as decision tree, random forest, KNN, boosting, and SVM, can also be used to classify other categories of surgical indicators. The Naive Bayes classification model could be used because the correlation coefficient between variables is low and the degree of correlation is not high. The classification accuracy, recall, and precision for KirkaldyWillis scores are high. Therefore, the Naive Bayes classification model is reasonable to classify the KirkaldyWillis scores.

\section{Data Availability}

The data used to support the findings of the study are available from the corresponding author upon request.

\section{Ethical Approval}

Between 2009 and 2014, the authors treated 37 consecutive patients with TST via a modified transfacet approach [7]. This study was approved by the Ethics Committee of Affiliated Hospital of Fujian Medical University.

\section{Conflicts of Interest}

The authors declare no conflicts of interest.

\section{Acknowledgments}

The authors sincerely thank the participants for their help and willingness to participate in this study. This study was supported by the National Natural Science Foundation of China (11601083 and U1805263), Natural Science Foundation of Fujian Province, China (2019J01451), Program for Probability and Statistics: Theory and Application (IRTL1704), and Innovative Research Team in Science and Technology in Fujian Province University (IRTSTFJ).

\section{Supplementary Materials}

The supplementary information files contain the table of patients treated in this study. (Supplementary Materials)

\section{References}

[1] C. S. Restrepo, R. Katre, and A. Mumbower, "Imaging manifestations of thoracic tuberculosis," Radiologic Clinics of North America, vol. 54, no. 3, pp. 453-473, 2016.

[2] Y. Yao, H. Zhang, M. Liu et al., "Prognostic factors for recovery of patients after surgery for thoracic spinal tuberculosis," World Neurosurgery, vol. 105, pp. 327-331, 2017.

[3] C. Fisahn, F. Alonso, G. A. Hasan et al., "Trends in spinal surgery for Pott's disease (2000-2016): an overview and bibliometric study," Global Spine Journal, vol. 7, no. 8, pp. 821-828, 2017.

[4] Y. Gao and Y. Ou, "Comparison between titanium mesh and autogenous iliac bone graft to restore vertebral height through posterior approach for the treatment of thoracic and lumbar spinal tuberculosis," PLoS One, vol. 12, no. 4, pp. 1-12, 2017.

[5] R. K. Jain and I. K. Dhammi, "Tuberculosis of the spine: a review," Clinical Orthopaedics and Related Research, vol. 460, pp. 39-49, 2007. 
[6] A. M. Quamar and S. A. Mir, "The Concept of evolution of thoracolumbar fracture classifications helps in surgical decisions," Asian Spine Journal, vol. 9, no. 6, pp. 984-994, 2015.

[7] Y. P. Huang, J. H. Lin, X. P. Chen et al., "Preliminary experience in treating thoracic spinaltuberculosis via a posterior modified transfacet debridement, instrumentation, and interbody fusion," Journal of Orthopaedic Surgery and Research, vol. 13, no. 292, pp. 1-9, 2018.

[8] J. S. Lee, K. P. Moon, S. J. Kim, and K. T. Suh, "Posterior lumbar interbody fusion and posterior instrumentation in the surgical management of lumbar tuberculous spondylitis," The Journal of Bone and Joint Surgery, vol. 89, no. 2, pp. 210-214, 2007.

[9] X. Q. He, Applied Regression Analysis (R Language Edition), Publishing House of Electronics Industry, pp. 55-84, Beijing, China, 2017.

[10] H. Li, Statistical Learning Method, Tsinghua University Publishing House, pp. 47-52, Beijing, China, 2012. 